\title{
DIRICHLET POLYHEDRA FOR CYCLIC GROUPS IN COMPLEX HYPERBOLIC SPACE
}

\author{
MARK B. PHILLIPS
}

(Communicated by Jonathan M. Rosenberg)

\begin{abstract}
We prove that the Dirichlet fundamental polyhedron for a cyclic group generated by a unipotent or hyperbolic element $\gamma$ acting on complex hyperbolic $n$-space centered at an arbitrary point $w$ is bounded by the two hypersurfaces equidistant from the pairs $w, \gamma w$ and $w, \gamma^{-1} w$ respectively. The proof relies on a convexity property of the distance to an isometric flow containing $\gamma$.
\end{abstract}

\section{INTRODUCTION}

In this paper we determine the shape of Dirichlet fundamental polyhedra for cyclic groups generated by a unipotent or hyperbolic element of $\mathbb{P} \mathbf{U}(n, 1)$ acting on complex hyperbolic $n$-space. We will see that these polyhedra have exactly two faces and that these faces have empty intersection. The proof relies on a convexity property of the distance to an isometric flow.

This fact is analogous to the visually obvious fact that the Dirichlet domain for a pure translation in Euclidean space is a two-sided strip whose sides are perpendicular to the line of translation. Fundamental domains of cyclic groups, however, are not always so simple. For example, the Dirichlet polygon centered at $(0,1)$ for the cyclic group acting on $\mathbb{R}^{2}$ generated by the map $(x, y) \mapsto$ $(x+1,-y)$ is a four-sided arrow-shaped polygon. In [7] Jørgensen describes a class of fundamental domains for cyclic groups acting in hyperbolic 3-space that have an unbounded number of sides. There are also examples of cyclic groups acting on Euclidean 3-space and real hyperbolic 4-space with infinitesided Dirichlet domains; see [2], for example.

Before beginning we describe our notation. For $z \in \mathbb{C}^{n}(M \in \mathrm{GL}(n, \mathbb{C}))$ we write $z^{\mathrm{T}}\left(M^{\mathrm{T}}\right)$ for the transpose and $z^{*}\left(M^{*}\right)$ for the conjugate transpose. When working in $\mathbb{C}^{n+1}$ we will write vectors and matrices in "block" form, for example, $Z=\left(z, z_{n+1}\right) \in \mathbb{C}^{n+1}$, where $z \in \mathbb{C}^{n}, z_{n+1} \in \mathbb{C}$, and

$$
\left[\begin{array}{lll}
A_{11} & A_{12} & A_{13} \\
A_{21} & a_{22} & a_{23} \\
A_{31} & a_{32} & a_{33}
\end{array}\right] \in \mathrm{GL}(n+1, \mathbb{C}),
$$

Received by the editors July 16, 1990.

1980 Mathematics Subject Classification (1985 Revision). Primary 53C55, 51M10, 51 M20.

Key words and phrases. Discrete groups, fundamental domain, complex hyperbolic space. 
where $A_{11}$ is an $(n-1) \times(n-1)$ matrix, $A_{12}, A_{13}, A_{21}^{\mathrm{T}}, A_{31}^{\mathrm{T}} \in \mathbb{C}^{n-1}$, and $a_{i j} \in \mathbb{C}$. The complement of a set $S$ will be denoted by $\widetilde{S}$.

\section{COMPLEX HYPERBOLIC SPACE}

Complex hyperbolic space $\mathbf{H}_{\mathbb{C}}^{n}$ is the unit ball in $\mathbb{C}^{n}$ with the Bergman metric $[3,5,6,8,10]$. It is the unique simply connected Kähler manifold of constant negative holomorphic sectional curvature -1 . The real sectional curvature varies between -1 and $-1 / 4$.

We shall work in the Siegel domain model of $\mathbf{H}_{\mathbb{C}}^{n}$. To obtain this model one starts with the Hermitian form on $\mathbb{C}^{n+1}$ of signature $(n, 1)$ given by

$$
\Psi(Z, W)=w^{*} z-\bar{w}_{n} z_{n+1}-\bar{w}_{n+1} z_{n},
$$

where $Z=\left(z, z_{n+1}\right)$ and $W=\left(w, w_{n+1}\right) \in \mathbb{C}^{n+1}$. Let $V_{-}=\left\{Z \in \mathbb{C}^{n+1} \mid\right.$ $\Psi(Z, Z)<0\}$, and let $\mathbb{P}$ be the usual projection operator on $\mathbb{C}^{n+1}-\{0\}$. $\Psi$ induces a Riemannian metric on $\mathbb{P}\left(V_{-}\right) ; \mathbf{H}_{\mathbb{C}}^{n}$ is $\mathbb{P}\left(V_{-}\right)$with this metric. If $Z \in V_{-}$we denote the corresponding point in $\mathbf{H}_{\mathbb{C}}^{n}$ by $[Z]$. In affine coordinates the Siegel domain model is represented by

$$
\left\{\left(z, z_{n}\right) \in \mathbb{C}^{n} \mid z^{*} z<2 \operatorname{Re}\left[z_{n}\right]\right\} .
$$

If $n=1$ this coincides with the right-halfplane model $\{\operatorname{Re}[z]>0\}$ for real hyperbolic two-space.

The Bergman metric in the Siegel domain is given by

$$
\cosh ^{2}\left(\frac{\rho([Z],[W])}{2}\right)=\frac{\Psi(Z, W) \Psi(W, Z)}{\Psi(Z, Z) \Psi(W, W)} ;
$$

$\rho([Z],[W])$ is the distance between $[Z],[W] \in \mathbf{H}_{\mathbb{C}}^{n}$.

\section{UNIPOTENT AND HYPERBOLIC ISOMETRIES}

The isometry group of $\mathbf{H}_{\mathbb{C}}^{n}$ is $\mathbb{P} \mathbf{U}(n, 1)$ acting by projective linear transformation. The nontrivial elements of $\mathbb{P} \mathbf{U}(n, 1)$ fall into three general conjugacy types, depending on the number and location of their fixed points. Elliptic elements have a fixed point in the interior of $\mathbf{H}_{\mathbb{C}}^{n}$. Parabolic elements have a single fixed point on the boundary of $\mathbf{H}_{\mathbb{C}}^{n}$. Loxodromic elements have exactly two fixed points on the boundary of $\mathbf{H}_{\mathbb{C}}^{n}$. This exhausts all possibilities; see [3], [1], or [5] for details.

A loxodromic element that is conjugate to an element in the identity component of $\mathbb{P O}(1,1) \subset \mathbb{P} \mathbf{U}(n, 1)$ is said to be hyperbolic. Loxodromic elements have an invariant geodesic-the geodesic connecting the two fixed points-and they act as translation along this geodesic. The hyperbolic elements are the ones that have no "twist" in the normal space to the invariant geodesic.

An element whose eigenvalues are all 1 is called unipotent. With the exception of the identity element, all unipotent elements are parabolic. Parabolic elements fix a point of $\partial \mathbf{H}_{\mathbb{C}}^{n}$. In the complement of this point in $\partial \mathbf{H}_{\mathbb{C}}^{n}$ they act in a way analogous to Euclidean rigid motions-each one is a translation followed by a rotation. The unipotent elements are the ones with no rotation component.

Any hyperbolic element is represented by a matrix that is conjugate to

$$
\left[\begin{array}{ccc}
I_{n-1} & 0 & 0 \\
0 & e^{-\frac{\tau}{2}} & 0 \\
0 & 0 & e^{\frac{\tau}{2}}
\end{array}\right]
$$


where $I_{n-1}$ is the $(n-1)$-dimensional identity matrix and $\tau \in \mathbb{R}$. This fixes the points $[0,0,1]$ and $\left[0,-\frac{1}{2}, 1\right] \in \partial \mathbf{H}_{\mathbb{C}}^{n}$ and translates by $\tau$ (complex hyperbolic) units along the geodesic between these points.

The unipotent elements fall into two subclasses: generic unipotents or principal parabolics and nongeneric unipotents or nonprincipal parabolics (see [6]). A principal parabolic is represented by a matrix conjugate to

$$
\left[\begin{array}{ccc}
I_{n-1} & 0 & \tau e_{1} \\
\tau e_{1}^{\mathrm{T}} & 1 & \frac{\tau^{2}}{2} \\
0 & 0 & 1
\end{array}\right],
$$

where $e_{1}=(1,0, \ldots, 0)^{\perp} \in \mathbb{C}^{n-1}$ and $\tau \in \mathbb{R}$.

A nonprincipal parabolic element is represented by a matrix conjugate to

$$
\left[\begin{array}{ccc}
I_{n-1} & 0 & 0 \\
0 & 1 & -i \tau \\
0 & 0 & 1
\end{array}\right]
$$

where $\tau \in \mathbb{R}$.

\section{EQUIDISTANT SURFACES}

Given two points $[Z]$ and $[W]$ in $\mathbf{H}_{\mathbb{C}}^{n}$, the equidistant surface of $[Z],[W]$ is the set

$$
S([Z],[W])=\left\{[X] \in \mathbf{H}_{\mathbb{C}}^{n} \mid \rho([X],[Z])=\rho([X],[W])\right\} .
$$

The locus of this equation decomposes as the disjoint union of a collection of complex linear subspaces along a real curve in $\mathbf{H}_{\mathbb{C}}^{n}$; see [9] for details. Because of this decomposition Mostow has termed these equidistant surfaces spinal hypersurfaces, where the spine is the real curve that determines the surface.

In [3] it is shown that the only totally geodesic submanifolds of $\mathbf{H}_{\mathbb{C}}^{n}$ are isometrically embedded copies of $\mathbf{H}_{\mathbb{C}}^{m}$ and $\mathbf{H}_{\mathbb{R}}^{m}$, where $m \leq n$. In particular, since there are no real codimension- 1 totally geodesic submanifolds, spinal surfaces are not totally geodesic.

\section{DiRICHLET POLYHEDRA}

Definition 5.1. Let $G$ be a subgroup of $\mathbb{P} \mathbf{U}(n, 1)$. A fundamental domain for $G$ is a connected open set $D$ of $\mathbf{H}_{\mathbb{C}}^{n}$ such that

(a) $\operatorname{vol}(\partial D)=0$ (Riemannian volume),

(b) there is a set $F$ with $D \subset F \subset \bar{D}$ such that $F$ contains exactly one point from each $G$-orbit in $\mathbf{H}_{\mathbb{C}}^{n}$.

This is a generalization of the definition that Beardon gives in [1] for groups of Möbius transformations.

One of the main uses of the fundamental domain is in studying the quotient manifold $\mathbf{H}_{\mathbb{C}}^{n} / G$. We would like the fundamental domain $D$ to serve as a "model" for the quotient manifold in the sense that $D / G$ should be homeomorphic to $\mathbf{H}_{\mathbb{C}}^{n} / G$. Based solely on Definition 5.1, however, this need not be the case; see $[1, \S 9.2]$ for an example. In order to guarantee this homeomorphism we must make one further assumption. 
Definition 5.2. A fundamental domain $D$ for $G$ is said to be locally finite if and only if each compact subset of $\mathbf{H}_{\mathbb{C}}^{n}$ meets only finitely many $G$-images of $\bar{D}$.

Theorem 5.1 [1]. A fundamental domain $D$ for $G$ is locally finite if and only if the map $\bar{D} \cap G(z) \mapsto G(z)$ is a homeomorphism of $\bar{D} / G$ onto $\mathbf{H}_{\mathbb{C}}^{n} / G$.

We now describe a construction that gives a locally finite fundamental domain for a given discrete group $G$. If $w$ is any point of $\mathbf{H}_{\mathbb{C}}^{n}$ that is not fixed by any element of $G$ other than $I$, then for each $g \in G(g \neq I)$ define

$$
L_{g}(w)=\left\{z \in \mathbf{H}_{\mathbb{C}}^{n} \mid \rho(z, w)=\rho(z, g w)\right\}
$$

and

$$
\begin{aligned}
H_{g}(w) & =\left\{z \in \mathbf{H}_{\mathbb{C}}^{n} \mid \rho(z, w)<\rho(z, g w)\right\} \\
& =\left\{z \in \mathbf{H}_{\mathbb{C}}^{n} \mid \rho(z, w)<\rho\left(g^{-1} z, w\right)\right\} .
\end{aligned}
$$

Note that $L_{g}(w)$ is a spinal hypersurface (not containing $w$ ) and that $H_{g}(w)$ is the halfspace that contains $w$ and is bounded by $L_{g}(w)$. In fact, $L_{g}(w)$ is the common boundary of $H_{g}(w)$ and $H_{g^{-1}}(g w)$.

Definition 5.3. The Dirichlet polyhedron $D(w)$ for $G$ with center $w$ is

$$
D(w)=\bigcap_{\substack{g \in G \\ g \neq I}} H_{g}(w) .
$$

$D(w)$ consists of the points of $\mathbf{H}_{\mathbb{C}}^{n}$ that are closer to $w$ than to any other point in the $G$ orbit of $w$. This is a special case of a general notion of the Dirichlet region of an arbitrary discrete subset. In [4] Ehrlich and Im Hof examine the topological and combinatoric aspects of these general Dirichlet regions.

Theorem 5.2. The Dirichlet polyhedron $D(w)$ is a locally finite fundamental domain for $G$.

This theorem is proved in [1] for for Fuchsian groups acting on $\mathbf{H}_{\mathbb{R}}^{2}$. The proof uses the fact that $D(w)$ is convex. Unfortunately Dirichlet polyhedra in $\mathbf{H}_{\mathbb{C}}^{n}$ need not be convex since the equidistant surfaces $L_{g}(w)$ are not totally geodesic. The Dirichlet polyhedra are, however, star-shaped (see [10]), and the proof of this theorem given in [1] applies just as well with "convex" replaced by "star-shaped."

\section{MAIN RESUlt}

We now examine the shape of Dirichlet polyhedra for cyclic groups generated by a unipotent or hyperbolic element $\gamma \in \mathbb{P} \mathbf{U}(n, 1)$. Such a $\gamma$ is conjugate to one of the forms (3.1), (3.2), or (3.3). The value of the real parameter $\tau$ in these forms is determined by $\gamma$; it is sometimes called the translation distance. Since $\tau$ will be fixed throughout our discussion, we will usually write $\gamma$ for $\gamma_{\tau}$. Note that $\gamma^{k}=\gamma_{\tau}^{k}=\gamma_{\tau k}$.

Our main result is the following. 
Theorem 6.1. Let $w \in \mathbf{H}_{\mathbb{C}}^{n}$ be any point. If $G$ is a cyclic subgroup of $\mathbb{P} \mathbf{U}(n, 1)$ generated by a unipotent or hyperbolic element $\gamma$, then the Dirichlet domain for $G$ centered at $w \in \mathbf{H}_{\mathbb{C}}^{n}$ has exactly 2 faces, and these faces do not intersect.

Theorem 6.1 will follow from a convexity property of the distance function along orbits of $G$.

Definition 6.2. A function $f: \mathbb{R} \rightarrow \mathbb{R}$ is convex if for every triple $a, b, c \in \mathbb{R}$ with $a<b<c$, the point $(b, f(b))$ on the graph of $f$ lies below the line segment connecting $(a, f(a))$ and $(c, f(c))$. Algebraically this is equivalent to

$$
a<b<c \Longrightarrow(c-a) f(b)<(c-b) f(a)+(b-a) f(c) \text {. }
$$

It is a well-known fact from elementary calculus that a twice differentiable function is convex if and only if its second derivative is positive.

Throughout the rest of this section let $w \in \mathbf{H}_{\mathbb{C}}^{n}$ be fixed and $H_{k}=H_{\gamma^{k}}(w)$. A point $z \in \mathbf{H}_{\mathbb{C}}^{n}$ is in $H_{k}$ if and only if $\rho(z, w)<\rho\left(z, \gamma^{k}(w)\right)$. Since $x \mapsto$ $\cosh ^{2}(x / 2)$ is an increasing function of $\mathbf{x}$, this is equivalent to $\cosh ^{2}(\rho(z, w) / 2)$ $<\cosh ^{2}\left(\rho\left(z, \gamma^{k}(w)\right) / 2\right)$. So if $f_{z}(k)=\cosh ^{2}\left(\rho\left(z, \gamma^{k}(w)\right) / 2\right)$, then $H_{k}$ consists of those points $z$ for which $f_{z}(0)<f_{z}(k)$.

Lemma 6.1. Suppose that for each $z \in \mathbf{H}_{\mathbb{C}}^{n}, f_{z}(k)$ is a convex function of $k$. Then the Dirichlet domain for $G$ centered at $w$ is the intersection of the two halfspaces $H_{1}$ and $H_{-1}$. Furthermore, the boundaries $L_{1}=\partial H_{1}$ and $L_{-1}=$ $\partial H_{-1}$ are disjoint.

Proof. Suppose $z \in H_{1}$, so that $f_{z}(0)<f_{z}(1)$. Applying (6.1) to $0<1<k$ yields

$$
\begin{gathered}
k f_{z}(1)<(k-1) f_{z}(0)+1 \cdot f_{z}(k) \\
=k f_{z}(0)-f_{z}(0)+f_{z}(k), \\
f_{z}(0)<k\left[f_{z}(0)-f_{z}(1)\right]+f_{z}(k)<f_{z}(k),
\end{gathered}
$$

which implies $z \in H_{k}$. Thus $H_{1} \subset H_{k}$ for $k>1$. The same argument with the convex function $k \mapsto f_{z}(-k)$ shows that $H_{-1} \subset H_{k}$ for $k<-1$. Therefore

$$
D(w)=\bigcap_{\substack{k=-\infty \\ k \neq 0}}^{\infty} H_{k}=H_{1} \cap H_{-1} .
$$

To prove the disjointness of the boundaries, note that $L_{i} \subset \widetilde{H}_{i}$, so it suffices to prove that $\widetilde{H}_{1} \cap \widetilde{H}_{-1}=\varnothing$. Now $z \in \widetilde{H}_{k}$ if and only if $f_{z}(0) \geq f_{z}(k)$; so if $z \in \widetilde{H}_{1} \cap \widetilde{H}_{-1}$, then

$$
f_{z}(0) \geq f_{z}(1) \text { and } f_{z}(0) \geq f_{z}(-1)
$$

Applying (6.1) to $-1<0<1$ yields

$$
\begin{gathered}
2 f_{z}(0)<f_{z}(-1)+f_{z}(1) \\
0<\left[f_{z}(-1)-f_{z}(0)\right]+\left[f_{z}(1)-f(0)\right],
\end{gathered}
$$

which implies that at least one of these last two summands is positive, contradicting (6.2). Therefore $\widetilde{H}_{1} \cap \widetilde{H}_{-1}=\varnothing$.

To prove the convexity of $f_{z}$ we will need the following fact about points in the Siegel domain (defined by equation (2.1)). 
Lemma 6.2. If $\left(z, z_{n}\right)$ and $\left(w, w_{n}\right)$ are (affine coordinates of) two points in the Siegel domain, then

$$
\operatorname{Re}\left[w^{*} z\left(w_{n}+\bar{z}_{n}\right)\right]<2\left(\left|z_{n}\right|^{2}+\left|w_{n}\right|^{2}\right) .
$$

Proof.

$$
\operatorname{Re}\left[w^{*} z\left(w_{n}+\bar{z}_{n}\right)\right] \leq\left|w^{*} z\right|\left|w_{n}+\bar{z}_{n}\right|
$$

By the Schwarz inequality,

$$
\leq \sqrt{z^{*} z} \sqrt{w^{*} w}\left|w_{n}+\bar{z}_{n}\right|
$$

and by $(2.1)$,

$$
\begin{aligned}
& \leq 2 \sqrt{\operatorname{Re}\left[z_{n}\right]} \sqrt{\operatorname{Re}\left[w_{n}\right]}\left|w_{n}+\bar{z}_{n}\right| \\
& \leq 2 \sqrt{\left|z_{n}\right|\left|w_{n}\right|}\left|w_{n}+\bar{z}_{n}\right| .
\end{aligned}
$$

Using $2\left|z_{n}\right|\left|w_{n}\right|<\left|z_{n}\right|^{2}+\left|w_{n}\right|^{2}$,

$$
\begin{aligned}
& \leq 2 \sqrt{\frac{\left|z_{n}\right|^{2}+\left|w_{n}\right|^{2}}{2}}\left|w_{n}+\bar{z}_{n}\right| \\
& =\sqrt{2} \sqrt{\left|z_{n}\right|^{2}+\left|w_{n}\right|^{2}}\left|w_{n}+\bar{z}_{n}\right| .
\end{aligned}
$$

Using $\left|w_{n}+\bar{z}_{n}\right|<\sqrt{2} \sqrt{\left|w_{n}\right|^{2}+\left|z_{n}\right|^{2}}$,

$$
\begin{aligned}
& \leq\left(\sqrt{2} \sqrt{\left|z_{n}\right|^{2}+\left|w_{n}\right|^{2}}\right)^{2} \\
& =2\left(\left|z_{n}\right|^{2}+\left|w_{n}\right|^{2}\right),
\end{aligned}
$$

which concludes the proof.

Lemma 6.3. Let $w \in \mathbf{H}_{\mathbb{C}}^{n}$, and suppose $\gamma \in \mathbb{P} \mathbf{U}(n, 1)$ is hyperbolic or unipotent. Then for each $z \in \mathbf{H}_{\mathbb{C}}^{n}, f_{z}(k)$ is a convex function of $k$.

Proof. Let $Z$ and $W$ be elements of $\mathbb{C}^{n, 1}$ that project to $z$ and $w$, respectively. $f_{z}(k)$ is then given by (2.2),

$$
f_{z}(k)=\frac{\Psi\left(Z, \gamma^{k}(W)\right) \Psi\left(\gamma^{k}(W), Z\right)}{\Psi(Z, Z) \Psi\left(\gamma^{k}(W), \gamma^{k}(W)\right)} .
$$

Since $\gamma$ preserves $\Psi$, the denominator here is a positive constant, so $f_{z}(k)$ is a positive constant multiple of the function

$$
g_{z}(k)=\Psi\left(Z, \gamma^{k}(W)\right) \Psi\left(\gamma^{k}(W), Z\right),
$$

and hence is convex if and only if this function is convex. Now note that $g_{z}$ can be extended from the integers to all of $\mathbb{R}$ by defining

$$
g_{z}(x)=\Psi\left(Z, \gamma_{x \tau}(W)\right) \Psi\left(\gamma_{x \tau}(W), Z\right),
$$

and we can check the convexity of this function by computing its second derivative. Furthermore, since convexity is unaffected by a linear change of scale ( $x \mapsto x / \tau)$, we can assume that $\tau=1$ and work with the function

$$
g_{z}(x)=\Psi\left(Z, \gamma_{x}(W)\right) \Psi\left(\gamma_{x}(W), Z\right)
$$


Suppose first that $\gamma=\gamma_{x}$ is hyperbolic. Then

$$
\gamma_{x}=\left[\begin{array}{ccc}
I_{n-1} & 0 & 0 \\
0 & e^{\frac{x}{2}} & 0 \\
0 & 0 & e^{\frac{x}{2}}
\end{array}\right]
$$

and

$$
g_{z}(x)=\left(w^{*} z-\bar{w}_{n} e^{-\frac{x}{2}}-z_{n} e^{\frac{x}{2}}\right)\left(z^{*} w-w_{n} e^{-\frac{x}{2}}-\bar{z}_{n} e^{\frac{x}{2}}\right) .
$$

A direct calculation shows that

$$
\frac{1}{2} g_{z}^{\prime \prime}(0)=2\left|z_{n}\right|^{2}+2\left|w_{n}\right|^{2}-\operatorname{Re}\left[z^{*} w\left(z_{n}+\bar{w}_{n}\right)\right],
$$

where of course $W=\left(w, w_{n}, 1\right)$ and $Z=\left(z, z_{n}, 1\right)$. By Lemma 6.2, this is positive, which implies that $g_{z}$ is convex.

Now suppose $\gamma$ is generic unipotent. Then

$$
\gamma_{x}=\left[\begin{array}{ccc}
I_{n-1} & 0 & x e_{1} \\
x e_{1}^{\mathrm{T}} & 1 & \frac{x^{2}}{2} \\
0 & 0 & 1
\end{array}\right]
$$

and

$$
\begin{aligned}
g_{z}(x)= & \left(\left(w+x e_{1}\right)^{*} z-x e_{1}^{\mathrm{T}} \bar{w}-\bar{w}_{n}-\frac{x^{2}}{2}-z_{n}\right) \\
& \times\left(z^{*}\left(w+x e_{1}\right)-\bar{z}_{n}-x e_{1}^{\mathrm{T}} w-w_{n}-\frac{x^{2}}{2}\right) .
\end{aligned}
$$

A computation yields

$$
\begin{aligned}
\frac{1}{2} g_{z}^{\prime \prime}(0) & =\left|w_{1}-\bar{z}_{1}\right|^{2}+\operatorname{Re}\left[w_{n}\right]-\operatorname{Re}\left[z^{*} w\right]+\operatorname{Re}\left[z_{n}\right] \\
& \geq\left|w_{1}-\bar{z}_{1}\right|^{2}+\operatorname{Re}\left[w_{n}\right]-\left|z^{*} w\right|+\operatorname{Re}\left[z_{n}\right] \\
& \geq\left|w_{1}-\bar{z}_{1}\right|^{2}+\operatorname{Re}\left[w_{n}\right]-\left|z^{*} z\right|\left|w^{*} w\right|+\operatorname{Re}\left[z_{n}\right] \\
& \geq\left|w_{1}-\bar{z}_{1}\right|^{2}+\operatorname{Re}\left[w_{n}\right]-2 \sqrt{\operatorname{Re}\left[w_{n}\right]} \sqrt{\operatorname{Re}\left[z_{n}\right]}+\operatorname{Re}\left[z_{n}\right] \\
& =\left|w_{1}-\bar{z}_{1}\right|^{2}+\left(\sqrt{\operatorname{Re}\left[w_{n}\right]}-\sqrt{\operatorname{Re}\left[z_{n}\right]}\right)^{2} \geq 0 .
\end{aligned}
$$

(Note that (2.1) implies $\operatorname{Re}\left[w_{n}\right]$ and $\operatorname{Re}\left[z_{n}\right]$ are positive, so $\sqrt{\operatorname{Re}\left[w_{n}\right]}{ }^{2}=$ $\operatorname{Re}\left[w_{n}\right]$ and $\sqrt{\operatorname{Re}\left[z_{n}\right]}{ }^{2}=\operatorname{Re}\left[z_{n}\right]$.)

The nongeneric unipotent groups are even easier. In this case,

$$
\begin{gathered}
\gamma_{x}=\left[\begin{array}{ccc}
I_{n-1} & 0 & 0 \\
0 & 1 & -i x \\
0 & 0 & 1
\end{array}\right], \\
g_{z}(x)=\left(w^{*} z-i x-\bar{w}_{n}-z_{n}\right)\left(z^{*} w+i x-w_{n}-\bar{z}_{n}\right),
\end{gathered}
$$

and it is easy to see that $g_{z}^{\prime \prime}(0)=2$.

Proof of Theorem 6.1. By Lemma 6.3, $f_{z}$ is convex for each $z$. Lemma 6.1 then implies that $D(w)$ has two faces, $L_{1}$ and $L_{-1}$, and that these faces do not intersect. 


\section{REFERENCES}

1. A. F. Beardon, The geometry of discrete groups, Graduate Texts in Math., vol. 91, SpringerVerlag, New York, Heidelberg, and Berlin, 1983.

2. B. H. Bowditch, Geometric finiteness for hyperbolic groups, Univ. of Warwick, preprint.

3. S. Chen and L. Greenberg, Hyperbolic spaces, Contributions to Analysis, Academic Press, New York, 1974, pp. 49-87.

4. Paul E. Ehrlich and Hans-Christoph Im Hof, Dirichlet regions in manifolds without conjugate points, Comment. Math. Helv. 54 (1979), 642-658.

5. D. B. A. Epstein, Complex hyperbolic geometry, Analytical and geometric aspects of hyperbolic space, (D. B. A. Epstein, ed.), London Math. Soc. Lecture Note Ser., vol. 111, Cambridge Univ. Press, Cambridge, 1987, pp. 93-111.

6. W. M. Goldman, Introduction to complex hyperbolic geometry, lecture notes for course at the Univ. of Maryland, January 1990.

7. Jørgensen, On cyclic groups of Möbius transformations, Math Scand. 33 (1973), 250-260.

8. S. Kobayashi and K. Nomizu, Foundations of differential geometry, II, Interscience Tracts in Pure Appl. Math., vol. 15, John Wiley \& Sons, New York, 1969.

9. G. D. Mostow, On a remarkable class of polyhedra in complex hyperbolic space, Pacific J. Math. 86 (1980), 171-276.

10. M. B. Phillips, Dirichlet polyhedra in complex hyperbolic space, Doctoral Dissertation, Univ. of Maryland at College Park, 1990.

Department of Mathematics and Computer Science, University of Richmond, RichMOND, VIRGINIA 23173

Current address: The Geometry Center, 1300 South Second St., Minneapolis, MN 55454

E-mail address: mbp@geom.umn.edu 